\title{
Characterization of rhodosaminyl-transfer by the
}

\author{
AknS/AknT glycosylation complex and its use \\ in reconstituting the biosynthetic pathway of
}

\author{
Aclacinomycin A
}

Catherine Leimkuhler, Micha Fridman, Tania Lupoli, Suzanne Walker, Christopher T. Walsh and Daniel Kahne*

Department of Chemistry and Chemical Biology, Harvard University,

Cambridge, Massachusetts 02138,

Department of Biological Chemistry and Molecular Pharmacology, Harvard Medical School, 240 Longwood Avenue, Boston, Massachusetts 02115, and

Department of Microbiology and Molecular Genetics, Harvard Medical School, Boston, Massachusetts 02115

kahne@chemistry.harvard.edu 


\section{Supporting Information-Experimental Procedures General Methods}

NMR spectra were recorded on a Varian Inova $400(400 \mathrm{MHz}$ for $1 \mathrm{H}, 100 \mathrm{MHz}$ for $13 \mathrm{C}$ and $162 \mathrm{MHz}$ for 31P) or a Varian Inova $500(500 \mathrm{MHz}$ for $1 \mathrm{H}$ and $125 \mathrm{MHz}$ for $13 \mathrm{C}$ ) or a Bruker DMX $500(500 \mathrm{MHz}$ for $1 \mathrm{H}, 125 \mathrm{MHz}$ for $13 \mathrm{C})$ instrument in the indicated solvent. Chemical shift are reported in units parts per million ( $\mathrm{ppm})$. 1H NMR spectra data are reported as follows: $\mathrm{CDCl} 3(7.26 \mathrm{ppm})$ or CD3OD (3.30 ppm) or D2O (4.80 $\mathrm{ppm})$. NMR spectra data are reported as follows: phosphoric acid $(0 \mathrm{ppm})$ was used as a external standard. Multiplicities are reported by using the following abbreviations $: \mathrm{s}=$ singlet, $\mathrm{d}=$ doublet, $\mathrm{t}=$ triplet, $\mathrm{q}=$ quartet, $\mathrm{m}=$ multiplet, $\mathrm{br}=$ broad, $J=$ coupling constant in Hertz.

Unless otherwise noted, all reactions were conducted under an argon atmosphere using anhydrous solvent (either distilled or passed through an activated alumina column). Commercially available reagents were used without further purification. Reactions were monitored by analytical HPLC were performed on a Hewlett-Packard 1100 series instrument using Phenomenex Luna $5 \mu \mathrm{m}$ C18 column $(250 \mathrm{~mm}$ x $4.6 \mathrm{~mm})$. Lowresolution mass spectra (LRMS) were obtained on an Agilent Technolgies LC/MSD instrument (Model \#G1956B) using electrospray ionization (ESI), while high-resolution mass spectra (HRMS), ESI mode, were obtained at the Harvard University Mass Spectrometry Facilities.

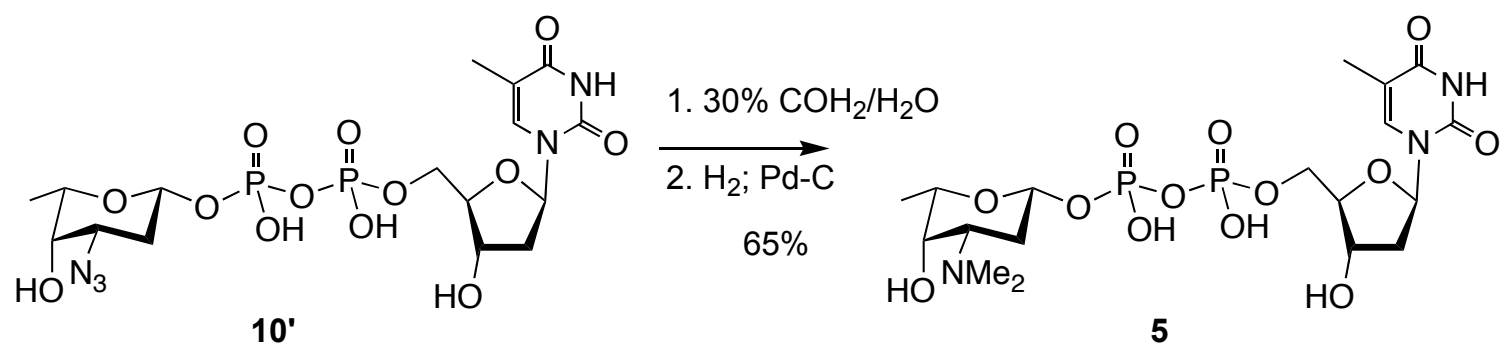

Thymidine 5'-( $\beta$-L-rhodosaminyl diphosphate) 5: Thymidine 5'-( $\beta$-L-3-azidodaunosaminyl diphosphate) ${ }^{1}$ (10', $\left.32 \mathrm{mg}, 57 \mu \mathrm{mol}\right)$ was dissolved in $\mathrm{H}_{2} \mathrm{O}(3 \mathrm{~mL})$, Palladium activated on carbon Deggusa type (40mg), and formaldehyde (36.5-38.0\% solution in water, $68 \mu \mathrm{L} \sim 0.85 \mathrm{mmol}$ ) was added. The mixture was stirred under a hydrogen balloon and monitoring was done by negative LR ESIMS and indicated completion after 14 hours (completion was determined by complete disappearance of the molecular peak of the starting material at $530.1[\mathrm{M}-\mathrm{H}]$ and the full conversion to the molecular peak of the product at $558.1[\mathrm{M}-\mathrm{H}]$. The reaction mixture was lyophilized, dissolved in a minimal volume of $1 \% \mathrm{NH}_{4} \mathrm{CO}_{3}$ water solution and purified by a sephadex LH-20 (Pharmacia) size exclusion chromatography. The fractions containing the product were lyophilized to give the ammonium salt of $5(20 \mathrm{mg}, 63 \%)$ as white foam. ${ }^{1} \mathrm{H}$ NMR $\left(500 \mathrm{MHz}, \mathrm{D}_{2} \mathrm{O}\right.$ ): ribose protons denoted as (') rhodosamine protons are donated as (") $\delta 7.59(\mathrm{~s}, 1 \mathrm{H}, 6-\mathrm{H}), 6.19\left(\mathrm{dd}, J_{1}=7.5, J_{2}=14.7 \mathrm{~Hz}, 1 \mathrm{H}, 1^{\prime}-\mathrm{H}\right), 5.09\left(\mathrm{dd}, J_{1}=8.1, J_{2}=\right.$ $10.3 \mathrm{~Hz}, 1 \mathrm{H}, 1 "-\mathrm{H}), 4.47$ (ddd, $\left.J=2.7,5.6,8.5 \mathrm{~Hz}, 1 \mathrm{H}, 33^{\prime}-\mathrm{H}\right), 4.02$ (m, 3H, 4'-H, 5'$\mathrm{H}(2 \mathrm{H})), 3.82$ (d, $J=3.4 \mathrm{~Hz}, 1 \mathrm{H}, 4 "-\mathrm{H}), 3.60$ (q, $J=6.5 \mathrm{~Hz}, 1 \mathrm{H}, 5 "-\mathrm{H}), 3.35$ (ddd, $J=3.4$, 7.51, $12.9 \mathrm{~Hz}, 1 \mathrm{H}, 3$ "-H), 2.77(s, 3H, N-Me 2 ), 2.74(s, 3H, N-Me $)$, 2.34 (m, 1H, 2"- $\mathrm{H}_{\mathrm{eq}}$ ), 2.15-2.26 (m, 2H, 2-H) 1.77 (s, 3H, thymidine $\left.C_{3}\right), 1.63$ (ddd, $J=10.1,10.6,12.4 \mathrm{~Hz}$, 
1H, 2"- $\mathrm{H}_{\mathrm{ax}}$ ), 1.09 (d, $\left.J=9.1 \mathrm{~Hz}, 3 \mathrm{H}, 6 "-\mathrm{H}\right) ;{ }^{31} \mathrm{P}$ NMR (162 MHz, $\left.\mathrm{D}_{2} O\right): \delta-10.62,-13.14$; HRMS (MALDI-TOF) for $\mathrm{C}_{18} \mathrm{H}_{31} \mathrm{~N}_{3} \mathrm{O}_{13} \mathrm{P}_{2}: 558.1230[\mathrm{M}-\mathrm{H}]$ calculated 558.1254.

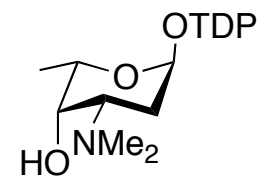

Thymidine 5'-( $\alpha$-l-rhodosaminyl diphosphate) 5 $\alpha$ : Thymidine 5'- $\alpha$-L-3-azidodaunosaminyl diphosphate $(52 \mathrm{mg}, 87 \mu \mathrm{mol})$ was prepared as was described for the preparation of compound 10' and dissolved in $\mathrm{H}_{2} \mathrm{O}(3 \mathrm{~mL})$. Palladium activated on carbon Deggusa type (40mg) and formaldehyde (36.5-38.0\% solution in water, $110 \mu \mathrm{L} \sim$ $1.38 \mathrm{mmol})$ were added to the solution. Yield- $33 \mathrm{mg} 68 \%{ }^{1} \mathrm{H}$ NMR $\left(500 \mathrm{MHz}, \mathrm{D}_{2} \mathrm{O}\right)$ : ribose protons denoted as (') rhodosamine protons are donated as (') $\delta 7.59(\mathrm{~s}, 1 \mathrm{H}, 6-$ H), 6.16 (dd, $J=7.0 \mathrm{~Hz}, 1 \mathrm{H}, 1 '-\mathrm{H}) 5.54$ (broad s, $J=9.3 \mathrm{~Hz}, 1 \mathrm{H}, 1$ "-H), 4.46 (ddd, $J=$ 3.2, 5.9, $\left.8.7 \mathrm{~Hz}, 1 \mathrm{H}, 3^{\prime}-\mathrm{H}\right), 4.09$ ( broad q, $\left.J=6.2,1 \mathrm{H}, 5^{\prime}-\mathrm{H}\right), 4.02$ (m, 3H, 4'-H, 5'-- ${ }_{2}$ ), 3.90 (broad s, 1H, 4"-H), 3.56 (ddd, $J=4.2,6.8,11.2 \mathrm{~Hz}, 1 \mathrm{H}, 3 "-\mathrm{H}), 2.78\left(\mathrm{~s}, 3 \mathrm{H}, \mathrm{N}-\mathrm{Me}_{2}\right)$, 2.74(s, 3H, N-Me $)$, 2.22(m, 3H, H2"-H $\left.\mathrm{H}_{\mathrm{eq}}, \mathrm{H}-2^{\prime}(2 \mathrm{H})\right), 2.37-2.31$ (m, 2H, 2-H ), 1.84 (m, $\left.1 \mathrm{H}, 2 "-\mathrm{H}_{\mathrm{ax}}\right), 1.77\left(\mathrm{~s}, 3 \mathrm{H}\right.$, thymidine $\left.C_{3}\right), 1.09(\mathrm{~d}, J=9.1 \mathrm{~Hz}, 3 \mathrm{H}, 6 "-\mathrm{H}) ;{ }^{31} \mathrm{P}$ NMR (162 $\mathrm{MHz}, \mathrm{D}_{2} O$ ): $\delta$-10.6, -12.9; HRMS (MALDI-TOF) for $\mathrm{C}_{18} \mathrm{H}_{31} \mathrm{~N}_{3} \mathrm{O}_{13} \mathrm{P}_{2} 558.1228[\mathrm{M}-\mathrm{H}]^{-}$ calculated $558.1254[\mathrm{M}-\mathrm{H}]$.

S 1. Oberthur, M.; Leimkuhler, C.; Kahne, D. Organic Letters 2004, 6, 2873-2876. 

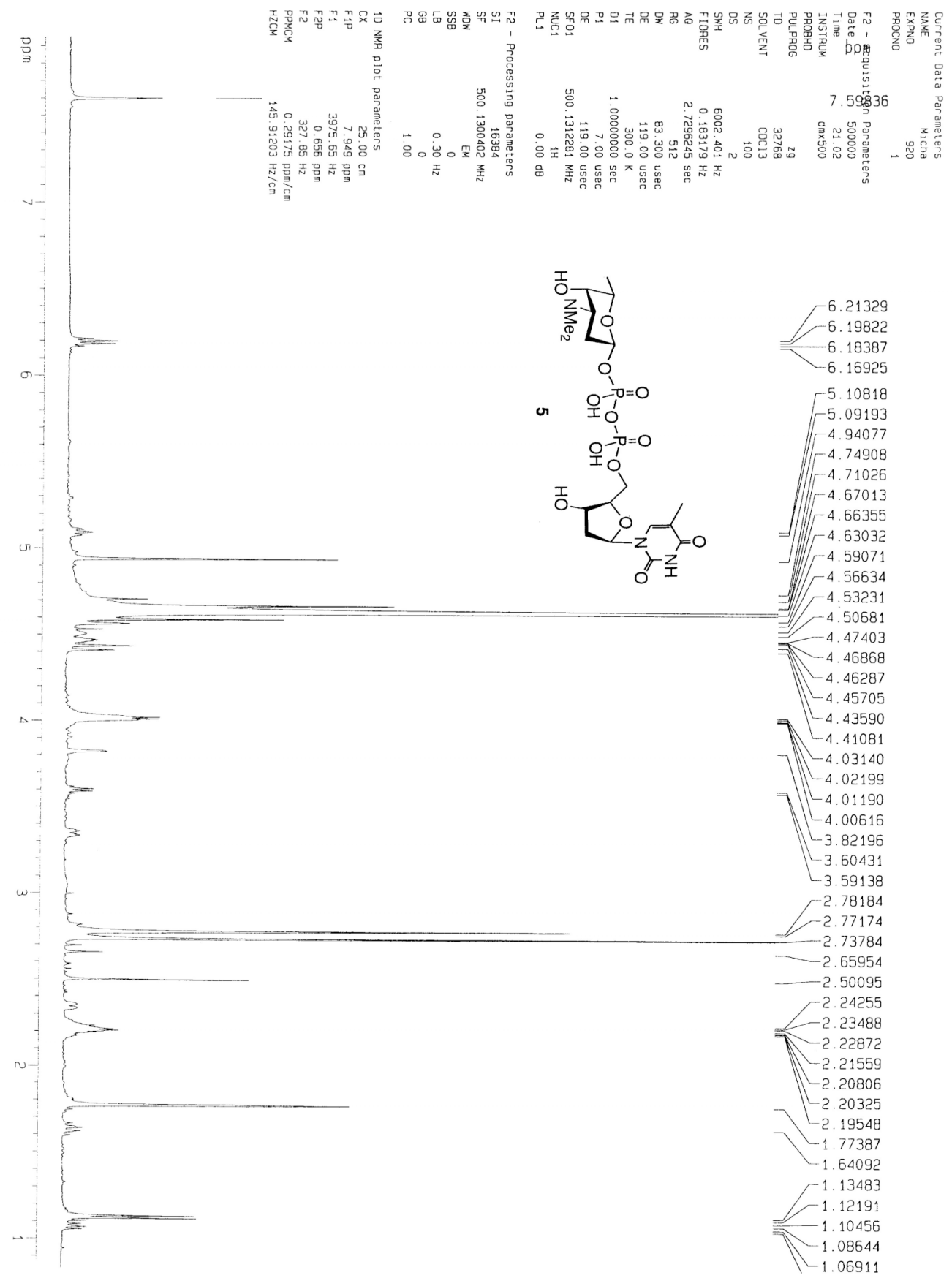

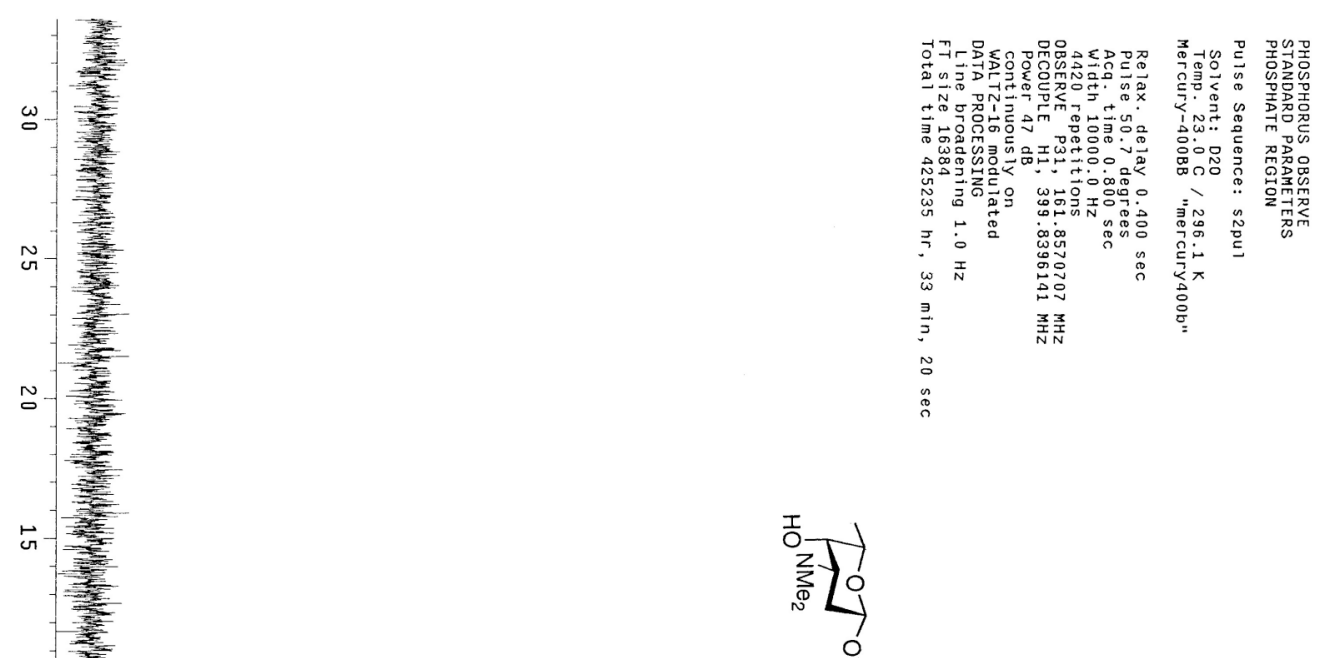

a 옴ำ $_{0}^{-0}=0$

옴ำ

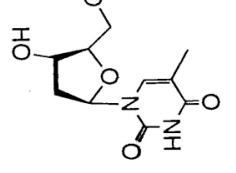

맙 출 